\title{
Use of a T-cell-based test for detection of tuberculosis infection among immunocompromised patients
}

\author{
F. Piana*, , L.R. Codecasa", P. Cavallerio*, M. Ferrarese", G.B. Migliori", \\ L. Barbarano ${ }^{+}$, E. Morra ${ }^{+}$and D.M. Cirillo*
}

ABSTRACT: The aim of this study was to compare the performance of the T-SPOT.TB ${ }^{\mathrm{TM}}$ test, a Tcell-based test, with the tuberculin skin test (TST) in the diagnosis of latent tuberculosis (TB) infection.

The study was carried out in 138 immunosuppressed haematology patients who had been nosocomially exposed to a case of smear-positive TB.

Overall, $44.2 \%$ of the contacts were positive by T-SPOT.TB ${ }^{\mathrm{TM}}$ test, and $17.4 \%$ by TST (concordance $67.8 \%$ ). The apparent prevalence of infection fell from 25.9 to $14.5 \%$ with the TST with increasing immunosuppression, although this difference was not significant. In contrast, the apparent prevalence of infection with the T-SPOT.TB ${ }^{\mathrm{TM}}$ test was unaffected at 44.6 and $44.3 \%$, respectively. The T-SPOT.TB ${ }^{\mathrm{TM}}$ test had an overall indeterminate rate of $4.3 \%$, and this was also unaffected by the level of immunosuppression.

This study suggests that the T-SPOT.TB ${ }^{\mathrm{TM}}$ test maintains its sensitivity and performance in immunocompromised patients, identifying a large number of truly infected patients anergic to the tuberculin skin test.

KEYWORDS: Contact tracing, haematology, interferon- $\gamma$, T-SPOT.TB ${ }^{\mathrm{TM}}$, tuberculosis

n January 2005, a male patient with multiple myeloma, attending the Haematology Chemotherapy Unit of Niguarda Hospital (Milan, Italy), was diagnosed with smear-positive pulmonary tuberculosis (TB). There was considerable concern over the exposure of other patients attending the same chemotherapy unit, since the index case had attended the Haematology Dept for $\sim 3$ months, with $\geqslant 55$ visits within this period.

As the risk of progression from latent to active TB amongst an immunosuppressed haematology patient group is higher than that of the general population [1, 2], it was very important to correctly identify those who had become infected. Unfortunately, the sensitivity of the current method of determining infection, the tuberculin skin test (TST), is known to be low [3,4]. Therefore, it was desirable to examine whether the new interferon (IFN)- $\gamma$-based tests for TB infection might give useful additional information, as the performance of these tests in immunosuppressed haematology patients is currently unknown [5].

For editorial comments see page 1 .
Of the two commercial tests currently available, the T-SPOT.TB ${ }^{\mathrm{TM}}$ assay (Oxford Immunotec, Oxford, UK), which enumerates individual activated TB-specific T-cells using enzyme-linked immunospot (ELISPOT) methodology, was chosen. This test was considered more useful than the QuantiFERON-TB Gold In Tube assay [6] since, at that time, only the T-SPOT.TB ${ }^{\mathrm{TM}}$ test had an internal positive control, and lymphocyte numbers and function were checked as part of the assay [7]. These two features were very important in the current setting because it was necessary to work with lymphocytes from people with underlying haematological diseases and who were undergoing chemotherapy, and thus it was necessary to know whether a negative result was a true negative or due to lack of function of T-cells. Moreover, the counting of Tcells, and thus the ability to correct for low lymphocyte counts, would give the best chance of getting a valid test result.

All the patients with possible nosocomial contact with the infectious patient were identified and underwent contact tracing. Contact tracing included clinical examination, a Mantoux test (TST), a T-SPOT.TB ${ }^{\mathrm{TM}}$ test and chest radiography.

\section{AFFILIATIONS}

*Emerging Bacterial Pathogens Unit, San Raffaele Scientific Institute for Research and Clinical Care,

"Villa Marelli Institute, Niguarda Ca' Granda Hospital,

${ }^{+}$Niguarda Ca' Granda Hospital, Milan, and

"WHO Collaborating Centre for Tuberculosis and Lung Disease, S Maugeri Foundation, Scientific Institute for Research and Clinical Care, Tradate, Italy.

CORRESPONDENCE

D.M. Cirillo

Emerging Bacterial Pathogens Unit

San Raffaele-Turro

Via Stamira D'Ancona 20

20127 Milan

Italy

Fax: 390226437989

E-mail: cirillo.daniela@hsr.it

Received:

September 212005

Accepted after revision:

February 272006 


\section{MATERIALS AND METHODS \\ Patient population}

All 138 patients (66 males, 72 females; mean (range) age 61 (1893) yrs; 136 Italians, two foreign-born) identified as nosocomial contacts of the index case were recalled for screening. The Ethical Committee of Niguarda Hospital (Milan, Italy) approved the study and patients gave their informed consent. Four patients had anamnesis of previously treated active TB disease and one had previously undergone isoniazid chemoprophylaxis for latent TB infection (LTBI). All were HIVnegative.

The haematological diseases were: non-Hodgkin's lymphoma (46; 33\%), multiple myeloma $(41 ; 30 \%)$, chronic lymphocytic leukaemia $(20 ; 14 \%)$, bone marrow dysplasia $(11 ; 8 \%)$, Hodgkin's lymphoma (10; 7\%), acute myeloid leukaemia (three; 2\%), acute lymphocytic leukaemia (one; $0.7 \%$ ), and chronic myeloid leukaemia (one; $0.7 \%$ ). Five (4\%) patients were treated for severe anaemia.

Mantoux testing was performed in January 2005, soon after the identification of the infectious case ( $>3$ months after first exposure), and T-SPOT.TB ${ }^{\mathrm{TM}}$ testing was performed 1530 days later.

\section{Tuberculin skin test screening}

The TST was performed according to international standards (5 TU of Siebert purified protein derivative of tuberculin (PPD) using BiocineTest-PPD (Chiron, Emeryville, CA, USA)) and indurations of $\geqslant 5 \mathrm{~mm}$ were considered positive, according to the Italian guidelines [8]. Nurses performing the TST were experienced in administering and reading the test, but were not blinded to the patient's history.

\section{T-SPOT.TB ${ }^{\text {TM }}$ test}

The T-SPOT.TB ${ }^{\mathrm{TM}}$ test was performed according to the manufacturer's instructions. Briefly, $8 \mathrm{~mL}$ blood were drawn into a BD Vacutainer ${ } \mathrm{CPT}^{\mathrm{TM}}$ tube (Becton Dickinson, Franklin Lakes, NJ, USA) and centrifuged to separate blood components. Lymphocytes were washed twice, the cell concentration adjusted and 250,000 cells seeded in each of four wells of the assay plate. The cells were stimulated for 16-20 h (under 5\% carbon dioxide at $37^{\circ} \mathrm{C}$ ) with media (nil control), phytohaemagglutinin (mitogen-positive control) or the specific antigens (6-kDa early secretory antigenic target and culture filtrate protein 10 , in separate wells). The IFN- $\gamma$ released by single cells was quantified using ELISPOT technology [4]. Results are presented as the number of spot-forming cells (SFCs). A positive antigen-specific result is defined as a well containing $\geqslant 6$ SFC more than the negative control.

The presence of a satisfactory reaction ( $>20 \mathrm{SFC}$ ) to the mitogen-positive control demonstrates T-cell function and also validates the assay result. An indeterminate result was reported when high background levels prevented interpretation or when $<20$ SFC were detected in the positive control wells.

\section{RESULTS}

At the time of screening, no signs of active TB were detected in the contacts by clinical examination and/or chest radiography. No patient has developed active TB 1 yr later.
In $16(11.6 \%)$ people, no TST result was obtained, due to either contraindications or nonreturns for reading.

Among the 138 patients tested by T-SPOT.TB ${ }^{\mathrm{TM}}$, it was not possible to collect sufficient T-cells in three $(2.1 \%)$ cases, and an indeterminate result was obtained in six (4.3\%) cases.

Valid T-SPOT.TB ${ }^{\mathrm{TM}}$ test results were, therefore, available for 129 patients and TST results for 122 .

Overall, $24(17.4 \%)$ subjects were positive by TST and 61 $(44.2 \%)$ by T-SPOT.TB ${ }^{\mathrm{TM}}$ test. The T-SPOT.TB ${ }^{\mathrm{TM}}$ result was positive in 34 TST-negative patients and the TST result was positive in three T-SPOT.TB ${ }^{\mathrm{TM}}$-negative patients (table 1 ). Concordance between the two tests (in the 115 patients for whom both test results were available) was $67.8 \% \quad(\kappa=0.34$, $\mathrm{p}<0.0001)$.

Bacille Calmette-Guérin (BCG) vaccination data were obtained for $84(60.9 \%)$ patients; only two were BCG vaccinated. This is consistent with the fact that BCG vaccination has never been extensively used in Italy. In both BCG-vaccinated patients, the TST result was positive and T-SPOT.TB ${ }^{\mathrm{TM}}$ test negative.

Details about the duration of contact were very difficult to collect as the only calculable data, in most cases, were the number of visits to the haematology unit coincident with the index case. Furthermore, it was not possible to classify the contacts by their closeness to the index case as the physical location of the patients during their visits is not routinely recorded. However, in 53 people with up to three visits coincident with the infectious case, the T-SPOT.TB ${ }^{\mathrm{TM}}$ test result was positive in $20(37.7 \%)$ subjects. Interestingly, this rate increased to $66 \%$ in the six patients with nine or more coincident visits with the infectious case. The difference between the two groups, however, did not reach significance due to the small number of patients. The Mantoux test showed no relationship to this one available measure of exposure, being negative in all of the patients in both of the above groups.

Results of white blood cell (WBC) counts were available for all patients. Seventy $(51.9 \%)$ patients had a pathological WBC count $\left(<4.3 \times 10^{3}\right.$ or $\left.>10.8 \times 10^{3} \mathrm{WBC} \cdot \mathrm{mm}^{-3}\right)$. Among these, there were two $(2.8 \%)$ indeterminate T-SPOT.TB ${ }^{\mathrm{TM}}$ test results due to lack of lymphocyte function. In the 65 patients with a normal count, there were four $(6.2 \%)$ indeterminate results,

\begin{tabular}{|c|c|c|c|c|c|}
\hline \multirow[t]{3}{*}{ TABLE } & \multicolumn{5}{|c|}{$\begin{array}{l}\text { Comparison of tuberculin skin test (TST) to } \\
\text { T-SPOT.TB }{ }^{\text {TM }} \text { test results in entire patient group }\end{array}$} \\
\hline & \multicolumn{3}{|c|}{ T-SPOT.TB ${ }^{\mathrm{TM}}$} & \multirow{2}{*}{$\begin{array}{l}\text { Insufficient } \\
\text { T-cells }\end{array}$} & \multirow[t]{2}{*}{ Total } \\
\hline & Positive & Negative & Indeterminate & & \\
\hline TST+ & 21 & $3^{\#}$ & 0 & 0 & $24(17.4)$ \\
\hline TST- & 34 & 57 & 5 & 2 & $98(71.0)$ \\
\hline No result & 6 & 8 & 1 & 1 & $16(11.6)$ \\
\hline Total & $61(44.2)$ & 68 (49.3) & $6(4.3)$ & $3(2.2)$ & 138 \\
\hline
\end{tabular}


showing that the extent of immunosuppression did not affect the T-SPOT.TB ${ }^{\mathrm{TM}}$ test's performance (nonsignificant). The T-SPOT.TB ${ }^{\mathrm{TM}}$ test was positive in $44.6 \%$ of those with normal WBC counts and in $44.3 \%$ of those with a pathological WBC count. In contrast, the level of positivity by TST fell from 25.9 to $14.5 \%$ between the same groups, although this fall did not quite reach significance $(\mathrm{p}=0.09$, Chi-squared test).

Stratifying the present population according to age, an increase in the rate of positive T-SPOT.TB ${ }^{\mathrm{TM}}$ tests with the age of the patients was found. In particular, in people aged $<60 \mathrm{yrs}$, $26.4 \%$ were positive, whereas this rate increased to $55.3 \%$ in people aged $>60$ yrs (odds ratio (OR) 3.17, 95\% confidence interval (CI) 1.48-6.79; $\mathrm{p}=0.0033)$. No such relationship was found with the TST (OR 1.54, 95\% CI 0.58-4.1; $\mathrm{p}=0.4816$ ).

\section{DISCUSSION}

On screening of 138 haematological patients who had been in contact with an infectious TB case, a significantly higher rate of positive T-SPOT.TB ${ }^{\mathrm{TM}}$ test results compared with TST screening was found. The T-SPOT.TB ${ }^{\mathrm{TM}}$ test was positive in $44.2 \%$ of the contacts versus $17.4 \%$ for the TST. It was important to determine whether the higher apparent prevalence of infection found with the T-SPOT.TB ${ }^{\mathrm{TM}}$ test was due to the TST being falsely negative due to anergy, or to the T-SPOT.TB ${ }^{\mathrm{TM}}$ test being falsely positive in a number of patients.

The present data, although clearly not definitive, support the former view that the T-SPOT.TB ${ }^{\mathrm{TM}}$ test was correctly identifying infected patients anergic to the TST. First, the results demonstrate that the T-SPOT.TB ${ }^{\mathrm{TM}}$ test result was not affected by the WBC counts of the patients, whereas the TST results were affected by increasing immunosuppression. The response to the positive control mitogen in the T-SPOT.TB ${ }^{\mathrm{TM}}$ assay provided a control against anergy (notwithstanding the fact that the indeterminate rate was only $4.3 \%$ overall, and lower in those with weaker immune systems). Secondly, the view that the T-SPOT.TB ${ }^{\mathrm{TM}}$ test gives false-positive results compared with the TST is not supported by the fact that, in routine contact tracing of nonimmunosuppressed close household contacts at the same institution, the reverse of what was found here was found, with the T-SPOT.TB ${ }^{\mathrm{TM}}$ test identifying less patients than the TST ( 54.5 versus $84.8 \%$ ) (data not shown). The clear differentiator in the present population was the widespread immunosuppression of all of the patients. Lastly, it is known that, in the Italian population, TST positivity correlates with age, presumably as a result of exposure when TB rates were higher than currently [9]. The fact that the T-SPOT.TB ${ }^{\mathrm{TM}}$ test result, and not that of the TST, was related to the age of the subjects further suggests that the T-SPOT.TB ${ }^{\mathrm{TM}}$ test was more closely reporting the true infection status of the patients than was the TST.

The current authors' tentative conclusions are supported by the substantial body of literature demonstrating that the T-SPOT.TB ${ }^{\mathrm{TM}}$ test result correlates more closely with exposure [10-14], is more sensitive than the TST [7, 15-18] and is not prone to cross-reaction to BCG and most nontuberculous mycobacteria [11, 19-20].

Based on the above considerations and additional clinical parameters, including the risk of side-effects, only the 61 T-SPOT.TB ${ }^{\mathrm{TM}}$-positive subjects were considered for chemoprophylaxis for LTBI (due to present or past exposure); eight patients aged $>75$ yrs were not treated (due to the high risk of side-effects), as well as the four patients with a previous history of $\mathrm{TB}$ and the patient with a history of chemoprophylaxis. The three TST-positive T-SPOT.TB ${ }^{\mathrm{TM}}$-negative patients were not treated. This decision was taken based on the fact that two of them were BCG vaccinated, whereas the third, an 18-yrold female with a Mantoux test result of $11 \mathrm{~mm}$, was exposed five times, but for very short periods, to the index case, as reported by the patient. It was considered more likely that these patients were falsely positive to the TST rather than falsely negative to the T-SPOT.TB ${ }^{\mathrm{TM}}$ test. False-negative T-SPOT.TB ${ }^{\mathrm{TM}}$ results can be excluded as the positive control demonstrated the presence of functional T-cells, even in the severely immunosuppressed patients.

None of the screened contacts has, after $1 \mathrm{yr}$, developed active TB.

Immunosuppressed groups are arguably the most important target for the screening and treatment of LTBI [21] because of their high risk of progression to active TB [2]. The poor performance of the TST in these groups greatly hinders this effort $[15,17]$. For this reason, there is great interest surrounding the use of new blood tests in the diagnosis of TB infection in immunosuppressed people [22] and whether they are more effective than the TST.

Recently, FERRARA et al. [23] reported that the QuantiFERONTB Gold assay was significantly adversely affected by immunosuppression, giving a high proportion of indeterminate results. Here, application of the T-SPOT.TB ${ }^{\mathrm{TM}}$ test for the LTBI screening of 138 haematological patients is reported. In these immunosuppressed individuals, the indeterminate rate of the T-SPOT.TB ${ }^{\mathrm{TM}}$ test was only $4.3 \%$, and the present authors believe that the T-SPOT.TB ${ }^{\mathrm{TM}}$ test demonstrated greater sensitivity than the TST, enabling the identification of 34 individual candidates for chemoprophylaxis.

To the present authors' knowledge, this is the first study demonstrating the utility of new blood tests in immunosuppressed non-HIV-positive patients. It suggests that the T-SPOT.TB ${ }^{\mathrm{TM}}$ test maintains sensitivity and performance in immunosuppressed patients (a finding recently confirmed in HIV patients [24]) and can identify infected patients anergic to the tuberculin skin test. Further investigation is required to prove whether the present observations can be corroborated by larger-scale studies.

\section{ACKNOWLEDGEMENTS}

The T-SPOT.TB ${ }^{\mathrm{TM}}$ kits were provided by Oxford Immunotec, Oxford, UK.

\section{REFERENCES}

1 American Thoracic Society, Centers for Disease Control and Prevention. Targeted tuberculin testing and treatment of latent tuberculosis infection. Am J Respir Crit Care Med 2000; 161: S221-S247.

2 Centers for Disease Control and Prevention. Screening for tuberculosis infection in high-risk populations. MMWR Morb Mortal Wkly Rep 1995; 44: 1-42. 
3 Huebner RE, Schein MF, Bass JB. The tuberculin skin test. Clin Infect Dis 1993; 17: 968-975.

4 Pesanti EL. The negative tuberculin test. Tuberculin, HIV, and anergy panels. Am J Respir Crit Care Med 1994; 149: 1699-1709.

5 Dheda K, Udwadia ZF, Huggett JF, Johnson MA, Rook GAW. Utility of the antigen-specific interferon- $\gamma$ assay for the management of tuberculosis. Curr Opin Pulm Med 2005; 11: 195-202.

6 Mazurek GH, LoBue PA, Daley CL, et al. Comparison of a whole-blood interferon $\gamma$ assay with tuberculin skin testing for detecting latent Mycobacterium tuberculosis infection. JAMA 2001; 286: 1740-1747.

7 Meier T, Eulenbruch HP, Wrighton-Smith P, Enders G, Regnath T. Sensitivity of a new commercial enzyme-linked immunospot assay (T SPOT-TB) for diagnosis of tuberculosis in clinical practice. Eur J Clin Microbiol Infect Dis 2005; 24: 529-536.

8 Italian Ministry of Health. Guidelines for Tuberculosis Control. Article 115. Decree-Law March 31, 1998 No. 112. Rome, Italian Ministry of Health, 1998.

9 Moro ML, Malfait P, Salamina G, D'Amato S. Tuberculosis in Italy: available data and open questions. Epidemiol Prev 1999; 23: 27-36.

10 Lalvani A, Pathan AA, Durkan H, et al. Enhanced contact tracing and spatial tracking of Mycobacterium tuberculosis infection by enumeration of antigen-specific T cells. Lancet 2001; 357: 2017-2021.

11 Ewer K, Deeks J, Alvarez L, et al. Comparison of T-cellbased assay with tuberculin skin test for diagnosis of Mycobacterium tuberculosis infection in a school tuberculosis outbreak. Lancet 2003; 361: 1168-1173.

12 Richeldi L, Ewer K, Losi M, et al. T cell-based tracking of multidrug resistant tuberculosis infection after brief exposure. Am J Respir Crit Care Med 2004; 170: 288-295.

13 Shams H, Weis SE, Klucar $\mathrm{P}$, et al. Enzyme-linked immunospot and tuberculin skin testing to detect latent tuberculosis infection. Am J Respir Crit Care Med 2005; 172: 1161-1168.
14 Zellweger JP, Zellweger A, Ansermet S, de Senarclens B, Wrighton-Smith P. Contact tracing using a new T-cell-based test: better correlation with tuberculosis exposure than the tuberculin skin test. Int J Tuberc Lung Dis 2005; 9: 1242-1247.

15 Lalvani A, Pathan AA, McShane H, et al. Rapid detection of Mycobacterium tuberculosis infection by enumeration of antigen-specific T cells. Am J Respir Crit Care Med 2001; 163: 824-828.

16 Chapman AL, Munkanta M, Wilkinson KA, et al. Rapid detection of active and latent tuberculosis infection in HIVpositive individuals by enumeration of Mycobacterium tuberculosis-specific T cells. AIDS 2002; 16: 2285-2293.

17 Richeldi L, Ewer K, Losi M, et al. Early diagnosis of subclinical multidrug-resistant tuberculosis. Ann Intern Med 2004; 140: 709-713.

18 Liebeschuetz S, Bamber S, Ewer K, Deeks J, Pathan AA, Lalvani A. Diagnosis of tuberculosis in South African children with a T-cell-based assay: a prospective cohort study. Lancet 2004; 364: 2196-2203.

19 Behr MA, Wilson MA, Gill WP, et al. Comparative genomics of BCG vaccines by whole-genome DNA microarray. Science 1999; 284: 1520-1523.

20 Andersen P, Munk ME, Pollock JM, Doherty TM. Specific immune-based diagnosis of tuberculosis. Lancet 2000; 356: 1099-1104.

21 Horsburgh R. Priorities for the treatment of latent tuberculosis infection in the United States. N Engl J Med 2004; 350: 2060-2070.

22 Pai M, Lewinsohn DM. Interferon- $\gamma$ assays for tuberculosis, is anergy the Achille's heel? Am J Respir Crit Care Med 2005; 172: 519-521.

23 Ferrara G, Losi M, Meacci M, et al. Routine hospital use of a new commercial whole blood interferon- $\gamma$ assay for tuberculosis infection. Am J Respir Crit Care Med 2005; 172: 631-635.

24 Dheda K, Lalvani A, Miller RF, et al. Performance of a Tcell-based diagnostic test for tuberculosis infection in HIVinfected individuals is independent of CD4 cell count. AIDS 2005; 19: 2038-2041. 